\title{
An architectural map of the anaphase-promoting complex
}

\author{
Brian R. Thornton, ${ }^{1,3}$ Tessie M. Ng, ${ }^{1,3}$ Mary E. Matyskiela, ${ }^{2}$ Christopher W. Carroll, ${ }^{2}$ \\ David O. Morgan, ${ }^{2}$ and David P. Toczyski ${ }^{1,4}$ \\ ${ }^{1}$ Cancer Research Institute, Department of Biochemistry and Biophysics, University of California, San Francisco, San \\ Francisco, California 94115, USA; ${ }^{2}$ Department of Physiology, University of California, \\ San Francisco, California 94143-2200, USA
}

\begin{abstract}
The anaphase-promoting complex or cyclosome (APC) is an unusually complicated ubiquitin ligase, composed of 13 core subunits and either of two loosely associated regulatory subunits, Cdc20 and Cdh1. We analyzed the architecture of the APC using a recently constructed budding yeast strain that is viable in the absence of normally essential APC subunits. We found that the largest subunit, Apc1, serves as a scaffold that associates independently with two separable subcomplexes, one that contains Apc2 (Cullin), Apc11 (RING), and Doc1/Apc10, and another that contains the three TPR subunits (Cdc27, Cdc16, and Cdc23). We found that the three TPR subunits display a sequential binding dependency, with Cdc27 the most peripheral, Cdc23 the most internal, and Cdc16 between. Apc4, Apc5, Cdc23, and Apc1 associate interdependently, such that loss of any one subunit greatly reduces binding between the remaining three. Intriguingly, the cullin and TPR subunits both contribute to the binding of Cdh1 to the APC. Enzymatic assays performed with APC purified from strains lacking each of the essential subunits revealed that only $c d c 27 \Delta$ complexes retain detectable activity in the presence of $\mathrm{Cdh} 1$. This residual activity depends on the C-box domain of Cdh1, but not on the C-terminal IR domain, suggesting that the C-box mediates a productive interaction with an APC subunit other than Cdc27. We have also found that the IR domain of Cdc20 is dispensable for viability, suggesting that Cdc20 can activate the APC through another domain. We have provided an updated model for the subunit architecture of the APC.
\end{abstract}

[Keywords: APC; yeast; cullin; TPR; Cdh1; Cdc20]

Supplemental material is available at http://genesdev.org.

Received November 30, 2005; revised version accepted December 22, 2005.

Ubiquitin ligases (E3s) serve as critical regulators of metabolism, the cell cycle, DNA damage response, stress response, and receptor signaling. These enzymes catalyze the transfer of ubiquitin from a ubiquitin conjugase (E2) to a substrate, often resulting in substrate degradation via the proteasome. Ubiquitin ligases fall into three categories: the HECT domain ligases, the U-box ligases, and RING-finger ligases (Pickart and Eddins 2004). The RING-finger ligases can be further divided into those that act as single subunits and those that act as part of a multisubunit complex, typified by an associated cullin subunit (cullin-RING ligases). Two of the most heavily studied cullin-RING ligases are the anaphase-promoting complex (APC) and SCF, both of which are composed of multiple subunits and serve as important regulators of the cell cycle (Vodermaier 2004). The better character-

\footnotetext{
${ }^{3}$ These authors contributed equally to this work. ${ }^{4}$ Corresponding author.

E-MAIL toczyski@cc.ucsf.edu; FAX (415) 502-3179.

Article and publication are at http://www.genesdev.org/cgi/doi/10.1101/ gad.1396906.
}

ized is SCF, which is composed of a modular specificity factor and three core subunits: a RING-finger subunit, a cullin subunit, and a scaffolding subunit (Willems et al. 2004; Petroski and Deshaies 2005). Crystal structures of the complex show that SCF holds an associated E2 and substrate in close proximity (Zheng et al. 2002). Although the precise mechanism of SCF catalysis has yet to be determined, the structure has provided great insights into how the enzyme functions.

Structural studies of the APC have proven more of a challenge. The APC is a cullin-RING ligase that ubiquitinates key regulators of mitosis, such as the mitotic and $\mathrm{S}$-phase cyclins and the anaphase inhibitor securin $(\mathrm{Pe}-$ ters 2002; Passmore 2004). Studies of the APC have lagged behind SCF because of the size of the complex and the inability to reconstitute it from purified components. The APC is composed of 13 core subunits (Yoon et al. 2002) and is activated by either of two weakly associating subunits Cdc20 or Cdh1 (Schwab et al. 1997; Visintin et al. 1997). Eight of the 13 core subunits are essential in budding yeast (Sikorski et al. 1991; Yu et al. 1998; Zachariae et al. 1998b; Peters 2002), making their 
study more challenging. Insights into the functions of nonessential subunits have been gained by studying strains in which they have been deleted. Deletions of SWM1, APC9, or CDC26 result in partial loss of Cdc27 and Cdc16 from purified APC (Zachariae et al. 1998b; Schwickart et al. 2004). Deletion of DOC1, on the other hand, reduces substrate binding and therefore enzyme processivity, but otherwise leaves the enzyme intact (Carroll and Morgan 2002; Passmore et al. 2003).

Like SCF, the APC contains a conserved RING-finger subunit (Apc11) and cullin-domain subunit (Apc2) that are essential and have been shown to be sufficient for limited catalytic activity in vitro (Gmachl et al. 2000; Leverson et al. 2000; Tang et al. 2001). The budding yeast APC contains three essential subunits with tetratricopeptide repeats (TPRs): Cdc27, Cdc23, and Cdc16, one of which (Cdc27) has been implicated in binding the activating subunit Cdh1 (Vodermaier et al. 2003; Kraft et al. 2005). The functions of the remaining subunits are unknown.

APC activity is primarily regulated by the binding of the activating subunits, Cdc20 and Cdh1 (Zachariae et al. 1998a; Jaspersen et al. 1999; Kramer et al. 2000; Rudner and Murray 2000). Two domains are thought to play roles in the binding of these subunits to the APC: a short internal motif called the C-box (Schwab et al. 2001) and a C-terminal IR dipeptide (Vodermaier et al. 2003). Cdc20 and Cdh1 also contain WD40 repeats that bind directly to APC targets, and thus may serve as a bridge between enzyme and substrate (Ohtoshi et al. 2000; Burton and Solomon 2001; Hilioti et al. 2001; Pfleger et al. 2001; Schwab et al. 2001; Burton et al. 2005; Kraft et al. 2005). The TPR protein Cdc27 has been implicated as an important binding site for the IR dipeptide motif of these subunits. Peters and colleagues have shown that Cdc27 is capable of binding an IR-containing peptide derived from the $C$ terminus of Cdh1 (Vodermaier et al. 2003) and that Cdh1 cross-links directly to Cdc27 in vitro in an IR-dependent fashion (Kraft et al. 2005).

Previously, we showed that the only obligatory targets of the APC for cell cycle progression are securin and the B-type cyclins (Thornton and Toczyski 2003). By deleting the genes encoding securin (Pds1) and the S-phase cyclin Clb5, while overexpressing the CDK inhibitor Sicl, we constructed a yeast strain that is capable of proliferating in the absence of the APC. In the present work, we used this strain to study the impact of deleting each of the essential APC subunits on enzyme structure. We find that the largest subunit, Apc1, binds independently to two subcomplexes. One contains the three TPR subunits as well as Cdc26, while the other contains Apc2, Apc11, and Doc1. The subunits of the TPR subcomplex bind sequentially to Apc1, with Cdc23 being the most directly associated and $\mathrm{Cdc} 27$ the most peripheral. Apc4, Apc5, Apc1, and Cdc23 bind interdependently, such that loss of any one subunit disrupts association between the remaining three. Using APC purified from strains lacking individual subunits, we also find that both Cdc27 and Apc2 are required for full binding of Cdh1 to the APC. Finally, we present biochemical and genetic evidence for the importance of an additional domain or domains besides the IR dipeptide in activator function.

\section{Results}

The APC is composed of two separable subcomplexes

We previously described the construction of a strain of Saccharomyces cerevisiae in which the APC is rendered nonessential. In strains lacking Pds 1 and Clb5 and overexpressing Sic1 ( $p d s 1 \Delta c 1 b 5 \Delta$ SIC1 $\left.{ }^{10 X}\right)$, any of the genes encoding normally essential APC subunits can be deleted. We examined the composition of the APC in these strains to uncover the binding dependencies for each of the essential subunits. To this end, we fused the tandemaffinity purification tag (TAP tag) (Puig et al. 2001) to the C terminus of Apc1, the largest subunit of the complex, reasoning that it may serve as a scaffold for other subunits. The TAP tag allows the rapid two-step affinity purification of proteins, first by binding to IgG beads and then by calcium-dependent binding to calmodulin beads. The APC1-TAP gene was introduced into the Apc1 locus in an $\mathrm{Apc}^{+}$strain and in strains bearing deletions of each of the remaining seven essential APC subunits (apc2s, apc11 $1, c d c 27 \Delta, c d c 16 \Delta, c d c 23 \Delta, a p c 4 \Delta$, and apc5 5 ). Extracts from each of these strains were subjected to TAP purification, and the resulting proteins were separated on SDS-PAGE and identified by silver staining or immunoblotting.

A purification profile from a strain with a wild-type APC is illustrated in Figure 1A and B. Silver staining allowed the identification of Apc1-TAP, Apc2, Cdc16, Cdc27, Cde23, and a tight doublet containing Apc4 and Apc5 (which can be resolved on some gels), based on the mobility of these subunits (Zachariae et al. 1998b; C. Carroll and D. Morgan, unpubl.). We further used polyclonal antibodies to identify Apc2, Apc11, Cdc16, Cdc27, Cdc23, and Doc1 by immunoblotting (Fig. 1B). The Apc1-TAP subunit was visualized with an antibody against the CBP domain of the TAP tag.

APC purifications were first carried out on extracts from strains lacking each of the TPR subunits Cdc27, Cdc16, and Cdc23. Examination of the elution profile of APC purified from a $c d c 27 \Delta$ strain revealed a largely intact complex; of the subunits tested, only Cdc27 itself was missing (Fig. 1C). By comparison, cdc16s strains lacked both Cdc16 and Cdc27 (Fig. 1D), while cdc23s strains lacked all three TPR subunits (Fig. 1E). These data suggest that the TPRs form an extended subcomplex that associates with Apc1 through Cdc23, with Cdc27 being the outermost subunit.

Identical purifications were then conducted on extracts from strains containing deletions of all other essential subunits. Peak fractions from each purification were normalized to Apc1-TAP levels and analyzed by silver staining and immunoblotting (Fig. 2A,B). Silver staining of the $c d c 27 \Delta$ and $c d c 16 \Delta$ complexes confirmed the results from Figure $1 \mathrm{C}$ and D. In the $c d c 23 \Delta$ strains, silver staining revealed that in addition to the loss of Cdc16 and Cdc27, Apc4 and Apc5 no longer associated 
A

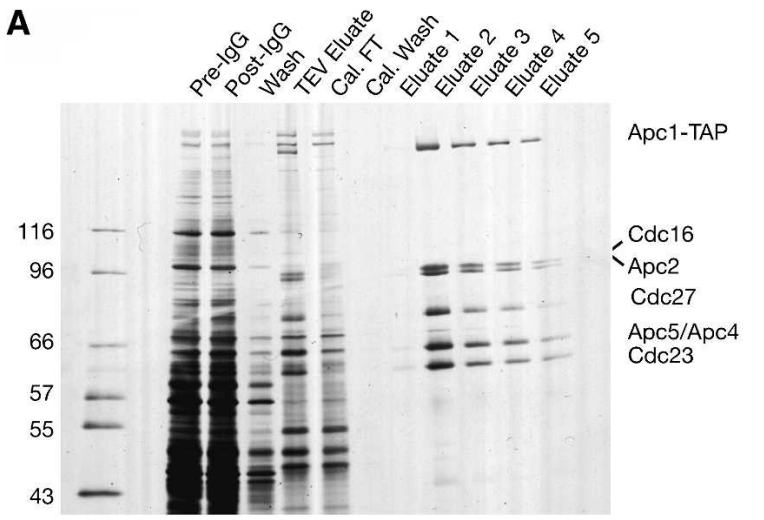

B

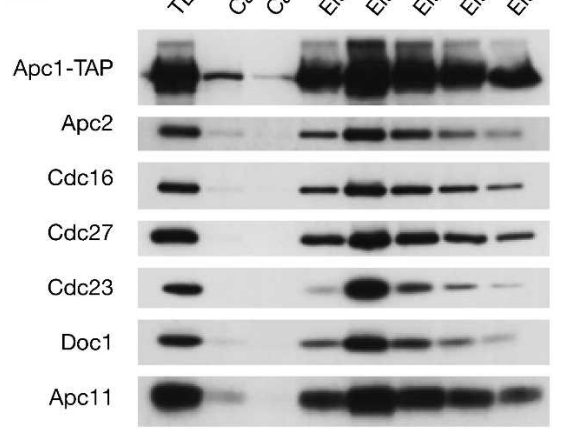

C

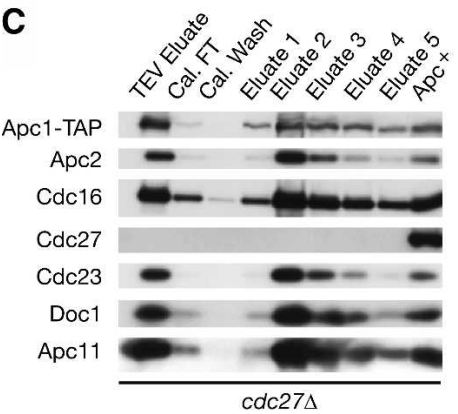

D

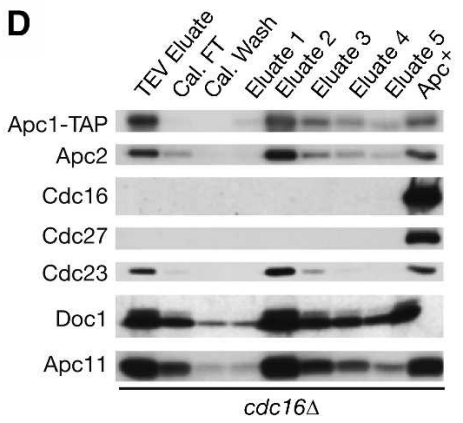

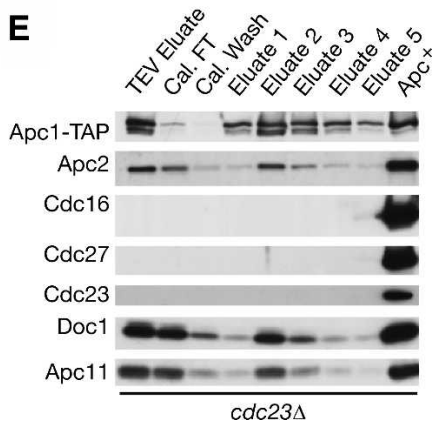

Figure 1. Purifications using Apc1-TAP allow the study of complexes lacking essential subunits. Asynchronous cultures of Apc ${ }^{+}$, $c d c 23 \Delta, c d c 16 \Delta$, and $c d c 27 \Delta$ strains carrying APC1-TAP were grown to log phase at $23^{\circ} \mathrm{C}$, harvested, and subjected to TAP purification. (A) $\mathrm{Apc}^{+}$purifications were resolved on a 7.5\%-15\% SDS-PAGE gradient gel and visualized by silver staining. Subunits were identified by size. $(B)$ Immunoblots of an $\mathrm{Apc}^{+}$Apc1-TAP purification. Samples were resolved on an SDS-PAGE gel and blotted with antibodies against the indicated proteins. $(C-E)$ Immunoblots of purifications from $c d c 27 \Delta, c d c 16 \Delta$, and $c d c 23 \Delta$ strains. The Apc ${ }^{+}$lane is from the "eluate 2" fraction of an Apc ${ }^{+}$Apc1-TAP purification.

with Apc1-TAP. Purifications from apc4s and apc5s strains similarly showed loss of all three TPR subunits (Fig. 2A,B). In addition, deletion of APC4 resulted in a reduction in the amount of Apc5 in the complex, and deletion of APC5 resulted in complete loss of Apc4 (Fig. 2A). This suggests that Cdc23 and Apc4 are mutually dependent on one another, and both are dependent on Apc5, for association with Apc1. Together the three subunits mediate binding of Cdc16 and Cdc27, in that order. We also observed that Cdc26, a small nonessential subunit, is lost specifically from those deletions that cause loss of Cdc16 (Fig. 2C).

In all of the above purifications, Apc2, Apc11, and Doc1 remained associated with Apc1-TAP. Deletion of APC11 resulted in the partial loss of Apc2 and Doc1. Purifications from apc2s strains, on the other hand, revealed the complete loss of Apc11 and Doc1 binding (Fig. 2B). Previous studies have shown that deletion of DOC1 does not affect the binding of Apc2, or any other subunit, to the APC (Carroll and Morgan 2002; Passmore et al. 2003). All other subunits examined remained associated in apc2 2 and apc11s purifications (Fig. 2B). These data suggest that Apc2 independently tethers Doc1 and Apc11. Apc1-TAP purified from cdc23s apc2s strains showed copurification of none of the other subunits tested.

The apparent loss of association we observed could, in fact, be due to reduced levels of one or more subunits in our deletion strains. To address this, we examined total protein levels of each of the subunits for which we have antibodies (Fig. 2D). In every case, we found that deletion of one of the essential subunits had no effect on the protein levels of the other subunits. We were unable to test the levels of Apc4 and Apc5 due to a lack of appropriate antibodies. For the subunits tested, however, we conclude that failure to copurify indicates reduced binding.

To address the role of Apc1 itself in subunit interactions, we introduced a tagged version of Cdc23 (Cdc23TAP) into $\mathrm{Apc}^{+}$and apc1s strains. Purifications were performed on these strains, and the proteins were analyzed by immunoblotting (Fig. 3A,B) and silver staining (Fig. 3C). The Cdc23-TAP purifications from apc1s strains retained associated Cdc16, Cdc27, and Cdc26, but no longer pulled down Apc2, Apc11, or Doc1 (Fig. $3 \mathrm{~A}-\mathrm{C})$. The presence of Apc4 and Apc5 could not be assessed because Cdc23-TAP comigrated with Apc4 and Apc5 in our SDS-PAGE gels (Fig. 3C). To eliminate this problem we tagged Apc4 or Apc5 with a triple Flag epitope in our Cdc23-TAP Apc ${ }^{+}$, Cdc23-TAP apc1s, and untagged strains. CDC23-TAP purifications successfully pulled down Apc4-3Flag and Apc5-3Flag from $\mathrm{Apc}^{+}$ strains, but not from apc1 1 strains (Fig. 3D,E), indicating that Apc4 and Apc5 require Apc1 to interact with Cdc23. 
Thornton et al.

A

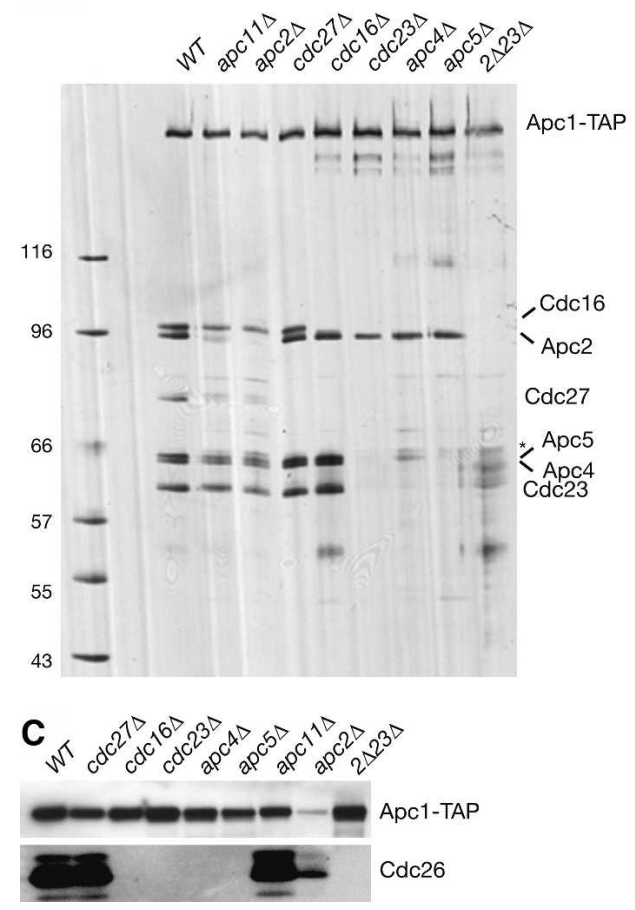

B
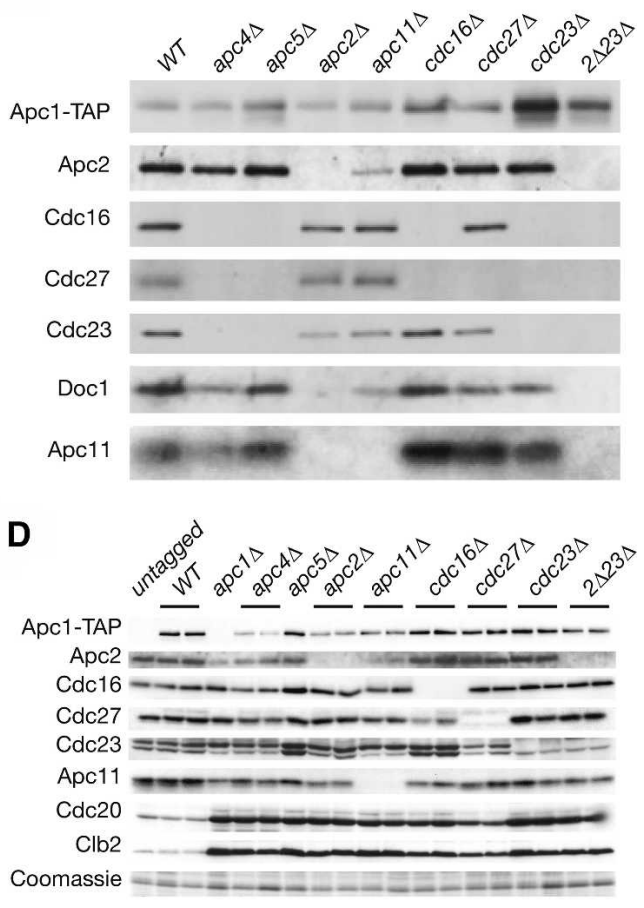

Figure 2. The TPR and cullin subunits are members of separable subcomplexes bound to Apc1. (A-C) Apc1-TAP purifications from $\mathrm{Apc}^{+}$strains and from each deletion strain were resolved on SDS-PAGE gels and visualized by silver stain $(A)$ or immunoblotting with the indicated antibodies $(B, C)$. Loading was normalized by Apc-TAP levels. $(D)$ Asynchronous cultures of strains bearing the indicated deletions were lysed directly into sample buffer, resolved on SDS-PAGE gels, and total protein was immunoblotted with the indicated antibodies. All strains except "untagged" and "apc1 $\Delta^{\prime}$ also carry APC1-TAP. The asterisk indicates a suspected breakdown product that appears just above the Apc5 band.

These data show that the TPR subunits and Cdc26 form a subcomplex and that this complex is stable in the absence of Apc1.

\section{Association of Cdh1 with the APC}

We have used our strains to analyze the effect of the deletion of each essential subunit on Cdh1 binding to the
APC. To accomplish this, we performed partial TAP purifications with strains bearing Apc1-TAP and lacking each of the essential subunits. After elution from IgG beads, the partially purified APC was bound to calmodulin beads and incubated with in vitro translated ${ }^{35}$ S-Metlabeled Cdh1. Beads were washed and analyzed to reveal the amount of APC-associated Cdh1. As a negative con-
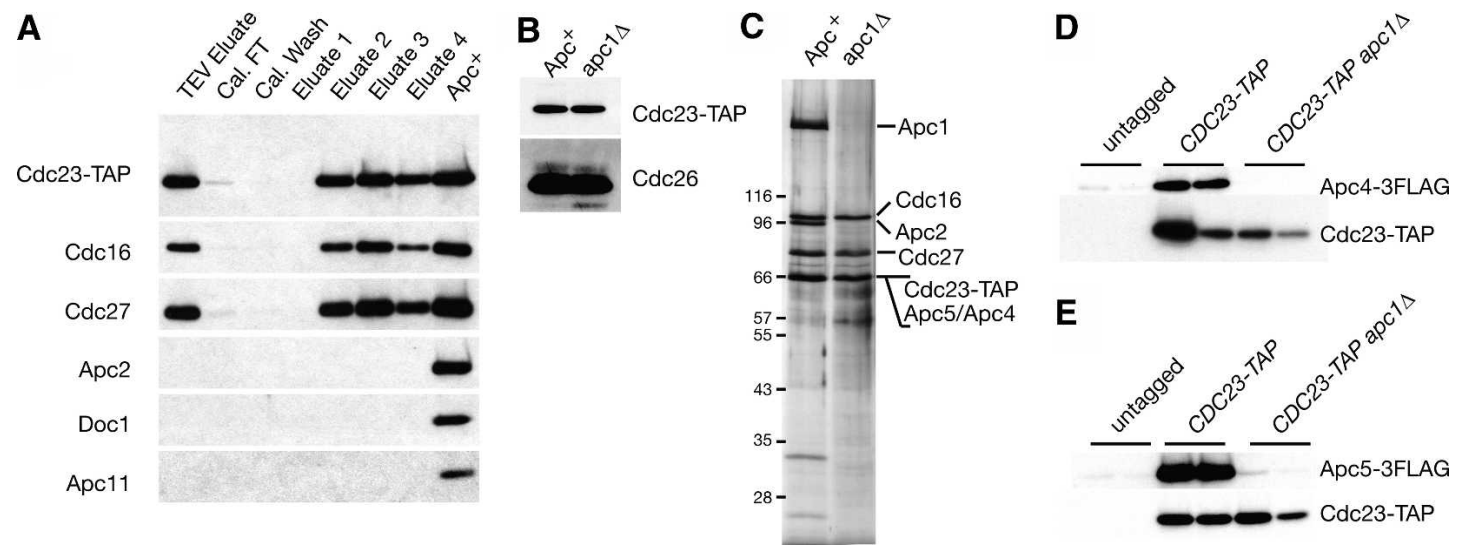

Figure 3. The TPR subunits form a stable subcomplex that includes Cdc27, Cdc16, Cdc23, and Cdc26. (A) SDS-PAGE gel and immunoblot of a Cdc23-TAP purification from an apc1s strain. Loading is as in Figure 1B. The "Apc " lane is the "eluate 2 " fraction from a Cdc23-TAP Apc ${ }^{+}$purification. (B) Cdc23-TAP purifications from $\mathrm{Apc}^{+}$and apc1s strains were normalized by Cdc23-TAP levels and blotted with antibodies against Cdc26. $(C)$ Cdc23-TAP purifications as in $B$ were resolved by SDS-PAGE and visualized by silver staining. $(D, E)$ Small-scale TAP purifications from untagged, CDC23-TA Apc ${ }^{+}$, or CDC23-TAP apc1D strains carrying either APC43Flag $(D)$ or APC5-3Flag (E). Two independent purifications are shown for each. 
trol, we used an extract from a strain lacking the TAP tag.

As shown in Figure 4A and B, wild-type APC bound as much as $12 \%$ of the input Cdh1. APC from $c d c 27 \Delta$ strains displayed greatly reduced Cdh1 binding, as recently reported by the Peters laboratory (Kraft et al. 2005) (Fig. 4A, cf. lanes 14-16 and 5-7). Complexes from $c d c 16 \Delta$ and $c d c 23 \Delta$ strains also showed no Cdh1 interaction. Surprisingly, APC from apc $2 \Delta$ strains showed a 10-fold reduction in Cdh1 association, despite the presence of Cdc27 in these complexes (Fig. 4A, cf. lanes 1113 and 5-7). APC from apc11s strains showed a two- to threefold decrease in Cdh1 binding (Fig. 4A, cf. lanes 8-10 and 5-7). However, the association of Apc2 with the APC was also reduced in apc11 strains. If one normalizes for the amount of Apc2 present (Fig. 4A, cf. lanes 5 and 10), apc11s and wild-type APC bind Cdh1 with equal efficiency. These data suggest that Apc2 is required for efficient Cdh1 binding to the APC.

It was recently reported that overexpression of an APC substrate (Hsl1) in vivo caused a twofold increase in the ability of Cdh1 to coimmunoprecipitate with Cdc16 (Burton et al. 2005). To examine the effects of substrate on Cdh1 binding to the APC, we added increasing amounts of a fragment of Hsl1 (667-872) to our binding assays. We did not detect an increase in Cdh1 binding to the APC in vitro when Hsll was present in $>1000$-fold excess over the concentration of Cdh1 (Supplementary Fig. 1). This result is consistent with a recent report from the Barford laboratory (Passmore and Barford 2005), where it was found that addition of substrate to an in vitro gel-shift assay did not result in increased Cdh1 binding to the APC.

We performed identical experiments using Cdc23-TAP to examine the TPR complex in either $\mathrm{Apc}^{+}$or apc1s strains. As seen with Apc1-TAP, Cdc23-TAP Apc ${ }^{+}$complexes associated with Cdh1 in our experiments (Fig. 4C, lanes 5-7). In contrast, the TPR complex purified from an apc1s strain failed to pull down Cdh1 above background levels, despite the presence all three TPR subunits (Fig. 4C, cf. lanes 8-10 and 5-7). We conclude that Cdc27 and Apc2 are each necessary, but not sufficient, for association of full-length Cdh1 with the APC in vitro.

\section{The C-box is required for binding to the APC}

Previous work suggested that the C-box motif of Cdh1 is important for its association with the APC (Schwab et al. 2001). We used our binding assay to determine whether a point mutant in a conserved C-box residue would eliminate APC binding.

We introduced a charge-swap mutation of a conserved C-box residue, R56D, into a GAL-CDH1-m11 vector (Zachariae et al. 1998a). This vector expresses a mutant form of Cdh1 that lacks its CDK phosphorylation sites; unphosphorylatable forms of Cdh1 lead to constant activation of the APC and therefore death (Zachariae et al. 1998a). In our $\mathrm{Apc}^{+}$strains, introduction of a $G A L$ -
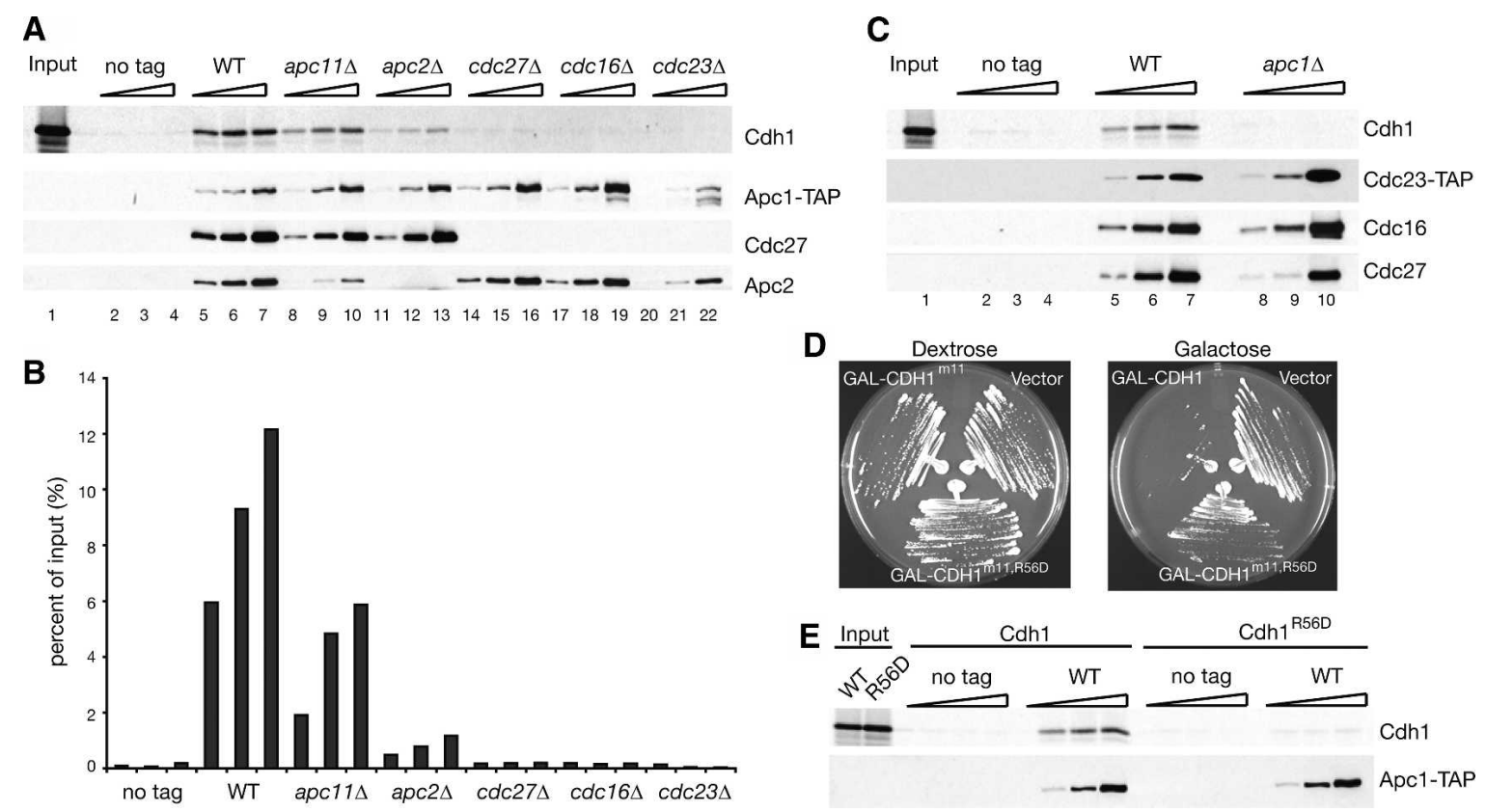

Figure 4. TPR and cullin subunits are required for full Cdh1 binding to the APC. (A) Apc1-TAP complexes were purified on IgG beads, eluted by TEV cleavage, bound to calmodulin beads in the presence of ${ }^{35} \mathrm{~S}$-Met-Cdh1, and eluted by the addition of EGTA. The three lanes for each strain represent $1 \times, 3.3 \times$, and $10 \times$ amounts of TEV eluate added to the calmodulin beads. Final eluates were transferred to a membrane and blotted with appropriate antibodies or exposed to a PhosphorImager. $(B)$ Quantitation of experiments done as explained in $A$. Data represent averages of two experiments. $(C)$ Binding experiments are as in $A$, but with Cdc23-TAP from Apc ${ }^{+}$or apc1s strains. (D) $\mathrm{Apc}^{+}$strains were transformed with blank vector, pGAL-CDH1 ${ }^{\mathrm{m} 11}$, or pGAL-CDH1 ${ }^{\mathrm{m} 11, \mathrm{R} 56 \mathrm{D}}$; streaked on plates containing dextrose or galactose; and grown at $23^{\circ} \mathrm{C}$. $(E)^{35} \mathrm{~S}$-Met-Cdh1 and ${ }^{35} \mathrm{~S}-$ Met-Cdh $1{ }^{\mathrm{R} 56 \mathrm{D}}$ were used in binding experiments performed as in $A$ on untagged and APC1-TAP $\mathrm{Apc}^{+}$strains. 
Thornton et al.

CDH1-m11 vector resulted in lethality on galactose (Fig. 4D). However, vectors expressing Cdh $1^{\text {R56Dm11 }}$ failed to kill the $\mathrm{Apc}^{+}$strain, implicating $\mathrm{R} 56$ as an important residue for $\mathrm{Cdh} 1$ function.

To address whether R56D has an effect on Cdh1 binding to the APC, we translated ${ }^{\mathrm{S} 35}$ Met-Cdh1 ${ }^{\mathrm{R} 56 \mathrm{D}}$ in vitro and tested its ability to bind wild-type complexes. Although the APC was capable of binding wild-type Cdh1, binding of $\mathrm{Cdh} 1^{\mathrm{R} 56 \mathrm{D}}$ was undetectable above background (Fig. 4E). Thus, R56D eliminates both Cdh1 binding to the APC in vitro and Cdh1 function in vivo.

\section{The APC retains activity in the absence of $C d c 27$}

We next determined whether any of the APC complexes lacking essential subunits still retained enzymatic activity. APC1-TAP strains carrying a deletion of each essential subunit were subjected to TAP purification, and the resulting complexes were subjected to a substrate ubiquitination assay using recombinant E1, E2, Cdh1, and ${ }^{35}$ S-Met-labeled Pds1 as a substrate. Of the mutant complexes, only $c d c 27 \Delta$ APC displayed detectable activity against Pds1 (Fig. 5A; data not shown). This activity was greatly reduced compared with wild type, but was still measurably higher than complexes lacking other subunits.

To examine further the residual activity of the $c d c 27 \Delta$ APC, we analyzed its activity in the presence of increasing amounts of Cdh1, using ${ }^{125} \mathrm{I}$-labeled sea urchin cyclin $\mathrm{B}$ as a substrate (Fig. 5B,C). Half-maximal activation of wild-type APC occurred at $29 \mathrm{nM} \mathrm{Cdh1}$, about the same concentration observed in our previous work /Carroll and Morgan 2002). The half-maximal Cdh1 concentration for the $c d c 27 \Delta \mathrm{APC}$ was clearly higher than this, but an accurate value could not be determined because we could not achieve a saturating concentration of Cdh1. Nevertheless, using curve-fitting analysis to predict

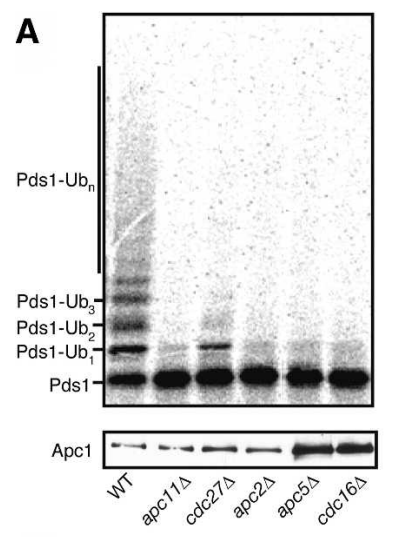

D
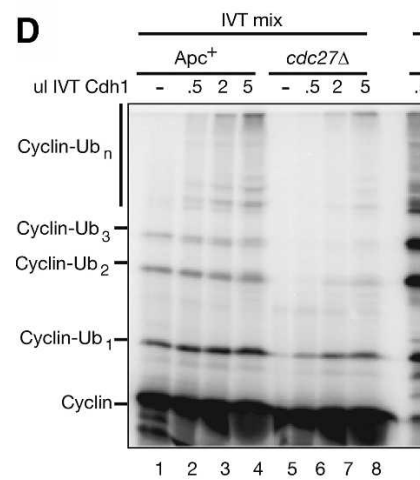

B

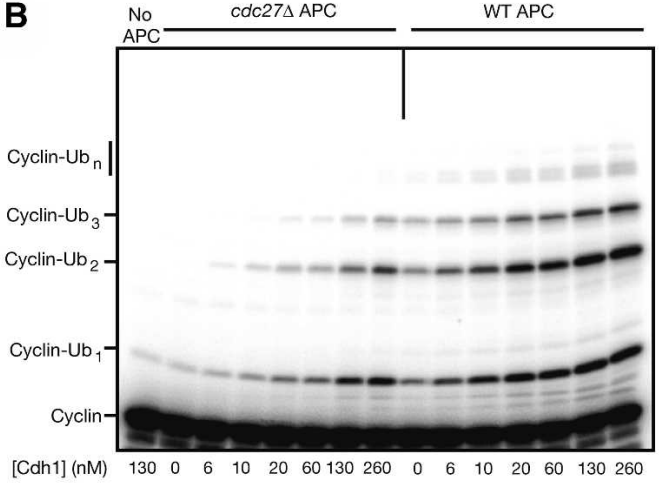

E $\frac{\text { WT Cdh1 }}{\frac{\mathrm{Apc}^{+}}{.5225} \quad \frac{\mathrm{cdc} 27 \Delta}{.525}}$

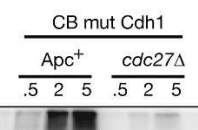

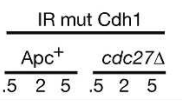

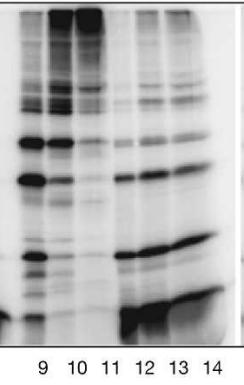

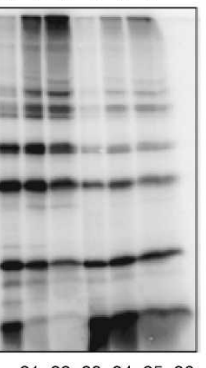

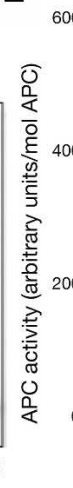

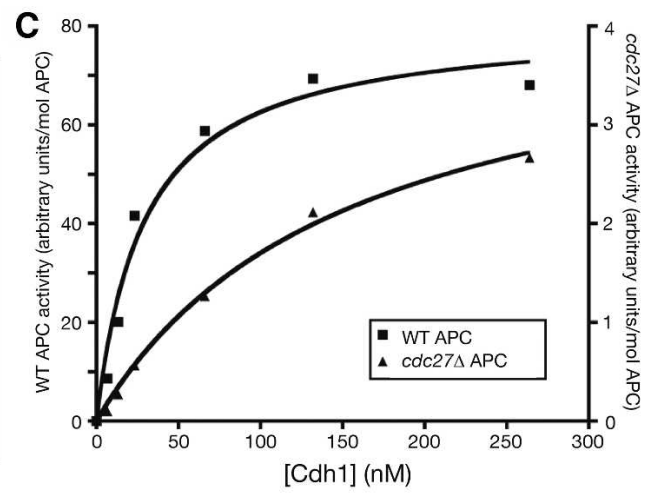

$\mathbf{F}$

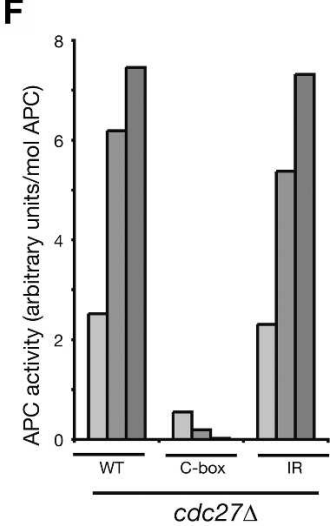

Figure 5. Cdh1 can activate the APC in the absence of Cdc27. (A) APC was immunoprecipitated from the indicated strain lysates by Apc1-TAP with IgG beads, and either used in a ubiquitination reaction with in vitro translated ${ }^{35}$ S-Met-Pds1 (top) or immunoblotted for Apc1-TAP levels (bottom). (B) Purified wild-type $(5 \mathrm{nM})$ and $c d c 27 \Delta$ APC $(60 \mathrm{nM})$ were incubated with the indicated amounts of purified 6His-Cdh1. Activity toward the ${ }^{125} \mathrm{I}$-labeled sea urchin cyclin B fragment was measured. $(C)$ Quantification of $B$ with a PhosphorImager and the ImageQuant program. Activity is normalized for enzyme concentration; note the different axes for wild-type and $c d c 27 \Delta$ activities. $(D)$ Wild-type and mutant Cdh1 were translated in vitro and added in the indicated amounts to wild-type and $c d c 27 \Delta$ APC reactions using ${ }^{125}$ I-labeled sea urchin cyclin B as a substrate. Reaction products were resolved on a $15 \%$ SDS-PAGE gel. Lanes 1-8 were incubated with IVT mix alone. No APC was added to the reactions in lanes 1 and 5. $(E, F)$ High-molecular-weight ubiquitin products $(>3$ ubiquitin adducts) were quantitated as in $C$, using a PhosphorImager and the ImageQuant program, and the counts were divided by the concentration of enzyme added. Graphs comparing wild-type APC $(E)$ and $c d c 27 \Delta$ APC $(F)$ are shown. Note that the scale of the axes for $E$ and $F$ differs because $c d c 27 \Delta$ APC activity is substantially lower than that of wild-type APC. APC activity is a measure of the amount of ubiquitin incorporation per unit of time per mole of APC. 
maximal cdc27 $\triangle$ APC activation, we estimate that halfmaximal stimulation of the $c d c 27 \Delta$ APC occurs at $\sim 150$ $\mathrm{nM}$ Cdh1, about fivefold higher than the concentration required for the wild-type enzyme. Thus, while Cdc27 is clearly important for normal Cdh1 binding to the APC (see also Fig. 4A), the ability of Cdh1 to stimulate $c d c 27 \Delta$ APC activity shows that productive Cdh1-binding sites must exist in the absence of Cdc27. It should also be noted that the considerable reduction in $c d c 27 \Delta$ APC catalytic activity is not simply the result of reduced Cdh1 association, since maximal $c d c 27 \Delta$ APC activity at near-saturating Cdh1 concentrations is much lower than maximal wild-type APC activity (note the scale of the respective axes in Fig. 5C). The low activity of the mutant enzyme may reflect additional roles for $\mathrm{Cdc} 27$ in catalysis.

Cdh1 contains two well-characterized APC interaction motifs, the C-box and the C-terminal IR dipeptide (Schwab et al. 2001; Vodermaier et al. 2003). One possible explanation for our results with the $c d c 27 \Delta \mathrm{APC}$ is that the IR motif binds to Cdc27 and the C-box binds to a second site. A prediction of this hypothesis is that the C-box would be particularly important for activity in $c d c 27 \Delta$ APC. We therefore measured the ability of C-box and IR mutants to activate wild-type and $c d c 27 \Delta$ APC. Because the C-box is essential for the Cdh1 APC interaction, we employed a Cdh1 C-box mutant (I58A P59A) that has only a mild defect in binding to wild-type APC (C. Carroll and D. Morgan, unpubl.). Cdh1 bearing either this C-box mutation or a deletion of the IR motif displayed a moderate defect in stimulating wild-type APC activity (Fig. 5D, cf. lanes 9 and 15,21, quantified in E). Deletion of the IR motif had little effect on the stimulation of $c d c 27 \Delta$ APC activity by Cdh1 (Fig. 5D, cf. lanes 12 and 24, quantified in F), as would be expected if Cdc27-dependent APC activity were mediated through the IR. The C-box mutant, on the other hand, was entirely unable to stimulate $c d c 27 \Delta$ APC activity (Fig. 5D, cf. lanes 12 and 18, quantified in F). These data strongly suggest that the C-box of Cdh1 mediates an interaction with the APC that is independent of the IR-Cdc27 interaction.

To confirm that $c d c 27 \Delta \mathrm{APC}$ retains activity in vivo, we tested the ability of overexpressed $\mathrm{Cdh} 1^{\mathrm{m} 11}$ to kill $c d c 27 \Delta$ strains. Overexpression of $\mathrm{Cdh} 1^{\mathrm{m} 11}$ is presumed to cause lethality by overactivating the APC, resulting in the continuous degradation of APC substrates. Strains that lack a functional APC should therefore be resistant to killing by GAL-CDH1-m11. We introduced either an empty vector or $p G A L-C D H 1-m 11$ into $\mathrm{Apc}^{+}, a p c 11 \Delta$, apc2 $\Delta$, and $c d c 27 \Delta$ strains, and tested their viability by spotting 10-fold dilutions on plates containing galactose (Fig. 6). Since Apc2 and Apc11 are thought to form the catalytic core of the APC, the enzyme should lack all activity and should be resistant to Cdh $1^{\mathrm{m} 11}$. Viability of $\mathrm{Apc}^{+}$strains was reduced by five orders of magnitude when plated on galactose, consistent with previously published results (Zachariae et al. 1998a). A similar loss of viability was observed in $c d c 27 \Delta$ strains. We also observed that total $\mathrm{Clb} 2$ and $\mathrm{Cdc} 20$ levels were lower in

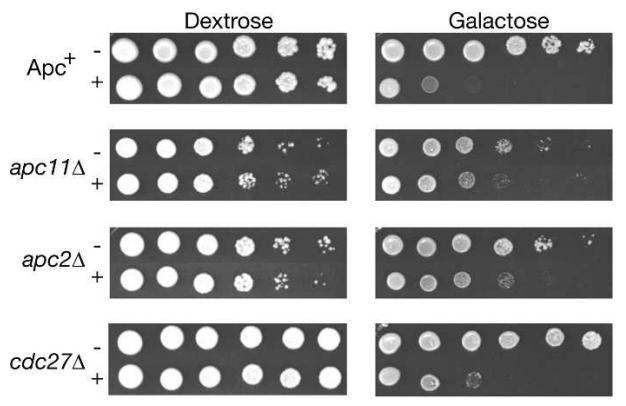

Figure 6. Overexpression of $\mathrm{Cdh} 1^{\mathrm{m} 11}$ kills $c d c 27 \Delta$ strains. $\mathrm{Apc}^{+}, a p c 11 \Delta, a p c 2 \Delta$, and $c d c 27 \Delta$ strains bearing an integrated GAL-CDH1 ${ }^{m 11}$ plasmid (+) or transformed with an empty CEN vector (-) were grown to mid-log and 10-fold dilutions were spotted on YEP plates containing either dextrose or galactose. Plates were grown at $23^{\circ} \mathrm{C}$.

$c d c 27 \Delta$ strains than in any other apcs strains (Fig. 2D), presumably because of residual $c d c 27 \Delta$ APC activity. These data strongly suggests that Cdh1 is capable of binding to and activating the APC through some subunit in addition to $\mathrm{Cdc} 27$.

Overexpression of $\mathrm{Cdh} 1^{\mathrm{m} 11}$ also caused some loss of viability in apc2 $\Delta$ and apc11s strains, although not as severe as the loss seen in $\mathrm{Apc}^{+}$and cdc27 $\Delta$ strains. This suggests that overexpression of Cdhl can affect cells independently of APC function, perhaps by binding to and sequestering substrates. This possibility has been suggested previously for the other activating subunit, Cdc20 (Clarke et al. 2003).

\section{The IR motif of Cdc20 is dispensable for viability}

To assess the impact of IR and C-box mutations on protein function in vivo, we chose to examine the effects of two mutations on the activating subunit Cdc20. This subunit is essential for viability, allowing us to test whether alleles bearing an R145D (in the C-box) or R610D (changing the C-terminal IR motif to ID) mutation were capable of supporting growth. Plasmids expressing wild-type $\mathrm{Cdc} 20, \mathrm{Cdc} 20^{\mathrm{R} 145 \mathrm{D}}$, or $\mathrm{Cdc} 20^{\mathrm{R} 610 \mathrm{D}}$ were transformed into $c d c 20 \Delta G A L-C D C 20$ strains and streaked on plates containing dextrose or galactose. Strains transformed with vector alone or with $c d c 20$ $R 145 D$ plasmids were unable to form colonies on plates containing dextrose (Fig. 7A), indicating that the C-box of Cdc20 is essential for its function. However, cdc20$R 610 D$ plasmids completely rescued the viability of cdc20 GAL-CDC20 strains on dextrose (Fig. 7A).

The ability of $c d c 20-R 610 D$ to sustain growth could be due to the particular point mutant used. We therefore used homologous recombination to generate $c d c 20-\Delta I R /$ $C D C 20$ heterozygous diploids, where one copy of CDC20 lacks the two codons for the C-terminal IR residues. The resulting tetrad dissection yielded predominantly four-spored tetrads, and the marker associated with $c d c 20-\Delta I R$ segregated 2:2 (Fig. 7B; data not shown). We assessed Cdc20 protein levels in the spores from two 

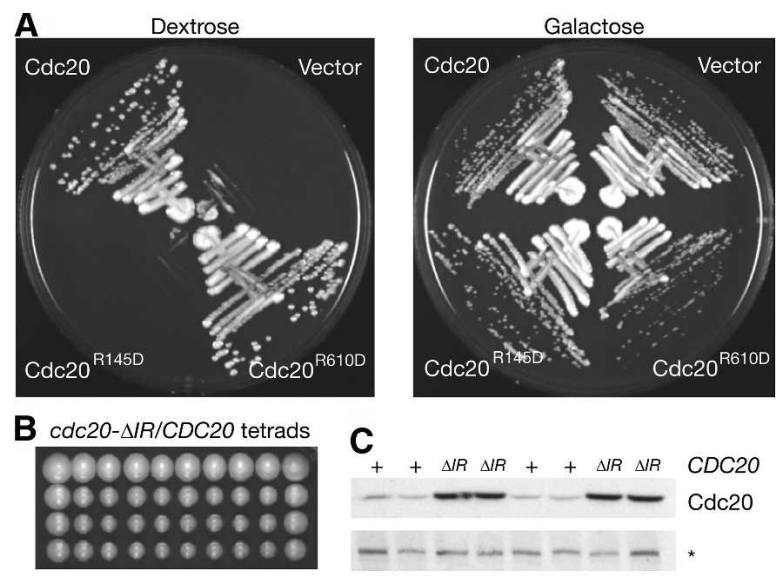

D

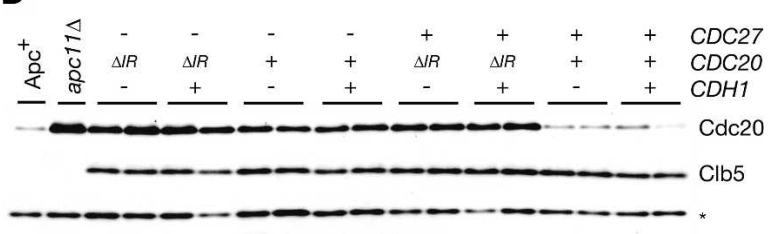

Figure 7. The IR domain of Cdc20 is dispensable for viability. (A) cdc20 $G A L-C D C 20$ strains transformed with empty vector or $2 \mu$ vectors carrying CDC20, cdc20-R145D, or cdc20-R610D were streaked on YEP + dextrose and YEP + galactose plates. $(B)$ Ten tetrads dissected from sporulated $c d c 20-\Delta I R / C D C 20$ heterozygous diploids. $(C)$ SDS-PAGE gel and immunoblot of two tetrads pictured in $A$, blotted with an $\mathrm{N}$-terminal Cdc20 antibody. (D) SDS-PAGE and immunoblot of strains with combinations of $c d c 27 \Delta, c d h 1 \Delta$, and $c d c 20-\Delta I R$ alleles. Two independent isolates are shown in each case. $\mathrm{Apc}^{+}$and apc11 $1 \Delta$ strains are included as controls, both of which are also pds1s clb5s $S I C 1^{10 X}$. In both $C$ and $D$, equivalent amounts of log-phase, asynchronous cultures were used, and the asterisk denotes a background band that serves as a loading control.

tetrads, and found that $\mathrm{Cdc} 20^{\Delta \mathrm{IR}}$ is expressed at higher levels than wild-type Cdc20 (Fig. 7C), suggesting that the loss of the IR motif results in Cdc20 stabilization.

We next assessed whether $c d c 20-\Delta I R$ viability was supported by overlapping Cdh1 function. Crosses against $c d h 1 \Delta$ strains revealed no synthetic lethality, yielding predominantly four-spored tetrads and many $c d c 20-\Delta I R$ $c d h 1 \Delta$ double mutants (data not shown). These mutants displayed no detectable growth defect, arguing that the IR motif of Cdc20 is dispensable for Cdc20 function even in the absence of Cdh1.

Stabilization of $\mathrm{Cdc} 20^{\Delta \mathrm{IR}}$ could be the result of a reduction in either Cdh1-mediated Cdc20 destruction or Cdc20 ubiquitination directly by the APC, possibly through Cdc27. To address this, we created strains with combinations of $c d c 20-\Delta I R, c d h 1 \Delta$, and $c d c 27 \Delta$ (all in the presence of the SIC1 ${ }^{10 X}$ allele [Thornton and Toczyski 2003] to support viability of $c d c 27 \Delta$ ), and examined Cdc20 levels from asynchronous cultures. Loss of Cdh1 had no impact on Cdc20 levels, but Cdc20 levels were indistinguishable between CDC20 cdc27A and cdc20- $\Delta I R$ CDC27 strains (Fig. 7D). Despite this dra- matic effect on Cdc20 levels, no detectable differences were seen in the levels of Clb5, a known target of Cdc20 (Fig. 7D). These data suggest that the IR motif of Cde20 serves primarily as a means for regulating Cdc20 levels through Cdc27 recognition. In addition, our observation that $c d c 20-\Delta I R \quad c d h 1 \Delta$ strains are viable in an otherwise wild-type strain, while $c d c 27 \Delta$ strains are inviable, indicates that Cdc27 plays an additional role in APC function beyond recognition of the IR motif.

\section{Discussion}

Apc1 is a bridge connecting two APC subcomplexes

In this study, we show that the largest APC subunit, Apc1, bridges two distinct subcomplexes (Fig. 8). The "catalytic core" subcomplex includes Apc2 and Apc11, the Cullin and RING-finger subunits that characterize the APC as a member of the multisubunit cullin-RING family of ubiquitin ligases. Our data also suggest that Doc1 associates directly with Apc2, since only deletion of APC2 eliminates Doc1 binding.

The second group of subunits contains the three TPR subunits Cdc27, Cdc16, and Cdc23, and requires Apc4 and Apc5 to interact with Apc1. We show here that the TPR subunits form a complex and that this complex remains assembled even in the absence of Apc1. Cdc26 is a nonessential, heat shock inducible subunit required to maintain full association of $\mathrm{Cdc} 27$ and $\mathrm{Cdc} 16$ at high temperatures (Zachariae et al. 1996, 1998b). Our data suggest that Cdc26 associates directly with Cdc16 and that loss of $\mathrm{Cdc} 27$ in a $c d c 26 \Delta$ strain may be an indirect effect of Cdc16 loss. Similarly, deletions of the nonessential subunits SWM1 and APC9 have been shown previously to cause a selective loss of Cdc27 and Cdc16 from the APC (Zachariae et al. 1998b; Schwickart et al.

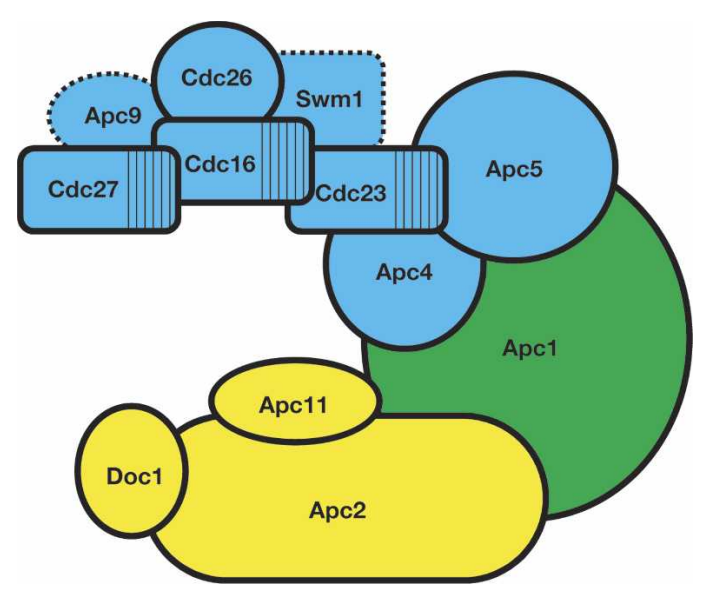

Figure 8. The APC is composed of two subcomplexes. Cdc23, Apc5, and Apc4 bind cooperatively to Apc1, which in turn binds to the more peripheral Cdc16, Cdc27, Cdc26, and likely Swm1 and Apc9. The catalytic subcomplex is composed of Doc1, Apc11, and Apc2, and depends on Apc2 for interaction with Apc1. Subunits not examined in this study are indicated with dotted lines. 
2004), suggesting that these proteins may be found in the TPR subcomplex as well (Fig. 8).

We have found that binding between Apc4, Apc5, $A p c 1$, and Cdc23 is interdependent, since the loss of any one subunit leads to a loss of association between the remaining subunits. Whether this reflects a shared binding interface between all four proteins or instead reflects conformational changes in the absence of certain binding partners remains to be elucidated.

It has previously been reported that the TPR subunits of the vertebrate APC can be removed from the complex by washing with high salt, leaving Apc4 and Apc5 associated with Apc1 (Vodermaier et al. 2003). It was also recently reported in budding yeast that temperature-sensitive mutations in Cdc23 cause loss of all three TPR subunits from the APC when grown at the restrictive temperature, but Apc4-TAP was still able to copurify with Apc1 (Schwickart et al. 2004). These results differ from our own results in $c d c 23 \Delta$ strains, where Apc4 and Apc5 appear to be entirely absent from Apc1-TAP complexes. This may indicate that $\mathrm{Cdc} 23$ is required for the assembly of Apc4 and Apc5 into a complex with Apc1, but is not required for the maintenance of this interaction.

The C terminus of Docl has been reported to interact with Cdc27 in vitro (Wendt et al. 2001). We show that Doc1 does not require any of the TPR subunits for association with the APC, but instead requires Apc2. This does not rule out the possibility that Doc1 interacts with Cdc27 or one of the other TPR subunits, but suggests that this interaction is relatively weak or occurs only under specific conditions. We discuss this further below.

\section{Cdh1 binding to and activation of the APC is mediated by both subcomplexes}

Regulation of APC activity is achieved through its two primary activating subunits, Cdc20 and Cdh1. Phosphorylation of Cdh1 blocks its ability to bind to the APC (Zachariae et al. 1998a; Jaspersen et al. 1999), while $\mathrm{Cdc} 20$ is regulated through its own APC-mediated degradation (Prinz et al. 1998; Shirayama et al. 1998), by the binding of inhibitory proteins such as Mad2 and Emi1 (Yu 2002; Tung and Jackson 2005), and by phosphorylation of the APC, which stimulates binding (Kramer et al. 2000; Rudner and Murray 2000). Since APC activation requires the binding of either Cdh1 or Cdc20, determining how and where they bind has been a topic of intense interest.

The C-terminal IR domain of Cdh1 has been suggested to bind directly to Cdc27 (Vodermaier et al. 2003; Kraft et al. 2005). Our results are consistent with this, as complexes lacking Cdc27 lose Cdh1 binding in vitro. However, while $c d c 27 \Delta \mathrm{APC}$ is far less active in ubiquitination assays in vitro, it does retain some Cdh1-dependent activity. This activity is independent of the IR motif of Cdh1, but is entirely dependent on a functional C-box. In addition, $c d c 27 \Delta$ strains are still highly sensitive to GAL-CDH1-m11, indicating that Cdh1 is still capable of activating the APC in the absence of Cdc27.
The simplest interpretation of these data is that the C-box of Cdh1 forms a productive interaction with an additional subunit besides $\mathrm{Cdc} 27$ and that neither association is absolutely essential. We show that loss of Apc2 results in an $\sim 10$-fold decline in Cdh1 binding in vitro despite the continued presence of Cdc27 (Fig. 4A, cf. Cdc27 levels in lanes 7 and 13). This suggests that a member of the cullin subcomplex is capable of a weak but productive interaction with the C-box of Cdh1. Alternatively, deletion of Apc2 may affect the ability of Cdc27 or other subunits to bind Cdh1. Further work will be required to test these possibilities.

Our data also suggest a role for Cde16 in $c d c 27 \Delta$ APC activity beyond Cdc27 association, since $c d c 16 \Delta$ APC lacks any measurable activity (Fig. 5A). Vodermaier et al. (2003) reported a weak interaction between Cdh1 C-terminal peptides and vertebrate Apc6, which is most closely related to yeast Cdc16. Our data suggest that the essential role of Cdc16 in APC function is not in binding the IR of Cdh1, since IR mutants retain activity, whereas APC from $c d c 16 \Delta$ strains do not. The Docl subunit of the APC contains a C-terminal IR dipeptide much like that of Cdh1 and Cdc20 (Vodermaier et al. 2003), and Doc1 has been implicated in substrate binding (Carroll and Morgan 2002; Passmore et al. 2003). Perhaps an interaction between the Doc1 IR domain and the TPR domain of Cdc16 creates a link between the two APC subcomplexes, and might cause the enzyme to partially close around a substrate and hold it in place once Cdh1 or Cdc20 bring it into proximity. This could explain how Docl affects the binding of a variety of substrates despite the lack of a WD40 propeller motif, a domain that has been shown to be critical for Cdh1 and Cdc20 to recognize substrates (Burton et al. 2005; Kraft et al. 2005).

We found that the $\mathrm{C}$-terminal IR residues of $\mathrm{Cdc} 20$ are dispensable for viability. Given the demonstrated importance of the IR motif in Cdh1 function (Kraft et al. 2005), this result suggests an important difference in the mechanisms by which Cdc20 and Cdh1 bind and activate the APC. Cdh1 and Cdc20 association with the APC is regulated in fundamentally different ways, where CDK phosphorylation of Cdh1 blocks association (Jaspersen et al. 1998; Zachariae et al. 1998a), while Cdc20 binding to the APC is stimulated by phosphorylation of the APC itself (Kramer et al. 2000; Rudner and Murray 2000). Earlier studies of Cdc20 stability have reported on a "destruction-box independent" mode of Cdc20 degradation that was still dependent on Cde23 and Cdc27 (Prinz et al. 1998). We speculate that the IR motif may be necessary for this destruction-box independent degradation, although it remains to be determined why the IR residues of Cdh1 do not result in a similar effect. However, we cannot rule out a positive role of Cdc20's IR motif in substrate targeting, since at least one temperature sensitive allele of an APC subunit, cdc23-1, is synthetically lethal with $c d c 20-\Delta I R$ (data not shown). Considerable additional study will be required to discern the role of the IR motif in either Cdh1 or Cdc20.

$\mathrm{Cdc} 27$ has been demonstrated to be the IR receptor for the APC (Vodermaier et al. 2003; Kraft et al. 2005), but 
our results indicate that $\mathrm{Cdc} 27$ plays an additional role in APC function. The CDC27 gene is essential, and while among the essential APC subunits only $c d c 27 \Delta$ APC retains activity, this residual activity is insufficient to sustain life in the absence of SIC $1^{10 X}$. If the function of $\mathrm{Cdc} 27$ were limited to being the IR receptor, we would expect $c d h 1 \Delta c d c 20-\Delta I R$ strains to be similarly inviable, yet they are viable and apparently healthy. Our analysis of $c d c 27 \Delta$ APC shows that the only APC subunit missing is Cdc27 itself (Fig. 1C), suggesting that the structure of the enzyme is largely intact. The simplest interpretation is that Cdc27 serves an additional function in catalysis besides activator binding.

In this study we present an architectural map of the APC and its activating subunits. The binding of the activating subunits appears to require multiple interactions. Different interactions might play a role during different steps of the formation of a polyubiquitin chain, possibly involving a handoff between a primary interacting subunit and multiple weakly interacting subunits. As it stands, most of the evidence about the requirements for activating subunit binding has been taken from the study of Cdh1, with the hope that it will extend to the related and less tractable Cdc20. However, our data suggest that while these adaptors encode similar interaction domains, these domains may serve a different purpose in each. Since Cdc20 binding is regulated in a fundamentally different manner than Cdh1, it may be that an entirely different set of dependencies will be observed for Cdc20. Our understanding of how the APC is activated may depend on discovering the differences, rather than the similarities, between its two most important regulatory subunits.

\section{Materials and methods}

\section{APC purification for structural studies}

A construct containing a TAP-tag and GAL-CDH1 ${ }^{\mathrm{m} 11}$ was integrated into $\mathrm{Apc}^{+}$and deletion strains at the $\mathrm{C}$ terminus of Apcl at its endogenous locus by homologous recombination. Asynchronous cultures were grown in YM1-dextrose at $23^{\circ} \mathrm{C}$, washed, and induced in media containing galactose for $10 \mathrm{~h}$ to late log phase. Twenty-gram to 30 -g pellets (wet weight) were collected and frozen down for future purification. TAP-tag purifications were performed as described previously (Puig et al. 2001) with the exception of the IPP150 buffer (20 mM HEPES at $\mathrm{pH} 8.0,150 \mathrm{mM} \mathrm{NaCl}, 0.2 \% \mathrm{NP} 40$ ) and the use of $10 \mathrm{mM}$ EGTA for the final elution step. Briefly, cells were lysed by grinding in a coffee grinder with dry ice and resuspended in 1.5 volumes of lysis buffer (IPP150 + 1 mM DTT and 5 mM EDTA and EGTA). Extracts were clarified and incubated with $300 \mu \mathrm{L}$ IgG beads (Amersham) at $4^{\circ} \mathrm{C}$ for $2 \mathrm{~h}$. Beads were then washed with $30 \mathrm{~mL}$ lysis buffer and $15 \mathrm{~mL}$ TEV cleavage buffer (IPP150+1 mM DTT and 0.5 mM EDTA). TEV cleavage was performed in $1 \mathrm{~mL}$ TEV cleavage buffer and $10 \mu \mathrm{L}$ TEV protease at $16^{\circ} \mathrm{C}$ for $2 \mathrm{~h}$. The TEV eluate was supplemented with $0.07 \% \beta M E, 1 \mathrm{mM}$ imidazole, $1 \mathrm{mM} \mathrm{MgAcetate}$, and $5 \mathrm{mM} \mathrm{CaCl}_{2}$ and added to $200 \mu \mathrm{L}$ calmodulin beads (Stratagene) and incubated overnight at $4^{\circ} \mathrm{C}$. Calmodulin beads were washed with $30 \mathrm{~mL}$ calmodulin binding buffer and eluted by stepwise addition of $200 \mu \mathrm{L}$ of elution buffer (10 mM EGTA). Final eluates were separated on a $7.5 \%$ $15 \%$ polyacrylamide gradient gel and visualized by silver staining. Loading was as follows: $1 / 1,000,000$ of pre-IgG, post-IgG, and wash samples; $1 / 60$ th of the TEV eluate, calmodulin flowthrough, and calmodulin wash samples, and $1 / 3$ of each elution fraction. Samples were also separated on $10 \%$ and $15 \%$ gels for subsequent immunoblotting. Loading amounts were as follows: $1 / 300,000$ of pre-IgG, post-IgG, and wash samples; $1 / 300$ of the TEV eluate, calmodulin flow-through, and calmodulin wash samples, and $1 / 20$ of each elution fraction.

\section{Cdh1-binding assay}

A TAP tag was integrated into wild-type and deletion strains at the $\mathrm{C}$ terminus of Apc1 at its endogenous locus by homologous recombination. Asynchronous cultures were grown in YM1dextrose at $23^{\circ} \mathrm{C}$, harvested, and frozen down. Pellet sizes varied depending on strains: $2 \mathrm{~g}$ for wild type, $4 \mathrm{~g}$ for $c d c 27 \Delta$ and $c d c 16 \Delta$, and $8.5 \mathrm{~g}$ for $a p c 2 \Delta, a p c 11 \Delta, c d c 23 \Delta$, and untagged wild type. TAP purifications were performed as above using scaled down but proportional volumes of reagents. Seventy-five microliters of IgG Sepharose beads (Amersham) were used for initial binding to extracts. Bound proteins were eluted into $500 \mu \mathrm{L}$ of TEV cleavage buffer. Eluates were then added at $1 \times, 3.3 \times$, and $10 \times$ concentrations to $2.5 \mu \mathrm{L}$ of calmodulin beads (Stratagene), $22.5 \mu \mathrm{L}$ of Sepharose 4B beads (Sigma), and $2.5 \mu \mathrm{L}$ of in vitro translated ${ }^{\mathrm{S} 35}$ Met-labeled Cdh1 or Cdh1 ${ }^{\mathrm{R} 56 \mathrm{D}}$ (TNT Quick Coupled Transcription/Translation Systems; Promega). Bound proteins were eluted off beads using buffer containing $20 \mathrm{mM}$ EGTA. Final eluates were separated by SDS-PAGE and analyzed by PhosphorImager (Molecular Dynamics) and immunoblotting.

Cdh1 $1^{m 11}$ overexpression assays

Asynchronous cultures were grown to log phase in YM1-dextrose and washed twice with YM1. Cells were then serially diluted 10-fold with YM1 and spotted onto a selective media plate containing either dextrose or galactose and grown at $23^{\circ} \mathrm{C}$ for $4 \mathrm{~d}$.

Construction of cdc20- $\Delta$ IR

The $c d c 20-\Delta I R$ allele was constructed by homologous recombination with a PCR product from primers sequence 5'-CGAGT GAGATTCATACAAGGAGGCCTCTAGTACCAGCCAATAT TTGTAGAGATTGTACTGAGAGTGCAC-3' and 5'-TACAT TATGTATGCGTGTATGGAAATTTCATTATATGCCTTGA CATGAACCTGTGCGGTATTTCACACCG-3', used to amplify the LEU2 marker from pRS305. Integration was confirmed by PCR with primers 5'-GGAGGTAATCCAGAGAATGC-3' and 5'-CACAGCAGAAGATGTTTAGC-3'.

\section{Ubiquitination assays}

Ubiquitination assays contain E1, E2, ubiquitin, ATP, Cdh1, the indicated substrate, and APC, and were performed as previously described (Carroll and Morgan 2002, 2005). For the immunoprecipitation activity assays in Figure 5A, cell lystates were prepared from the indicated strain by bead beating two times for 30 sec each in lysis buffer $(20 \mathrm{mM}$ HEPES at $\mathrm{pH} 7.4,150 \mathrm{mM} \mathrm{NaCl}$, $5 \mathrm{mM}$ EDTA, $0.2 \% \mathrm{NP}-40,50 \mathrm{mM} \beta$-glycerophosphate, $50 \mathrm{mM}$ $\mathrm{NaF}, 1 \mathrm{mM} \mathrm{Na}_{3} \mathrm{VO}_{4}, 1 \mathrm{mM}$ DTT, $1 \mathrm{mM}$ PMSF). APC was then bound to IgG Sepharose beads (Amersham), washed three times, and split into two halves. One half was incubated with E1, E2, ubiquitin, ATP, Cdh1, and in vitro translated ${ }^{35}$ S-labeled Pds1, 
for $1 \mathrm{~h}$ at room temperature before being run on a 7.5\% SDSPAGE gel and subsequently quantified with a Molecular Dynamics PhosphorImager (Amersham Biosciences) and the ImageQuant program. The other half was analyzed by immunoblotting for Apcl.

For the Cdh1 dose response assay in Figure 5B, wild-type and $c d c 27 \Delta$ APC were purified in parallel. The concentration of APC in the reactions was $\sim 5 \mathrm{nM}$ for wild-type APC and $60 \mathrm{nM}$ for $c d c 27 \Delta \mathrm{APC}$, and quantification of ubiquitination activity was normalized for the amount of APC present. The Cdh1 used in the assay was 6His-Cdh1 expressed in Sf9 cells with baculovirus and purified using metal affinity chromatography on cobalt resin. Although we previously referred to this protein as Hctl-His (Jaspersen et al. 1999), the tag is located at the $\mathrm{N}$ terminus of the protein. This Cdh1 was added in increasing amounts to APC reactions containing ${ }^{125}$ I-labeled sea urchin cyclin fragment (residues 13-110). After $20 \mathrm{~min}$ at room temperature, reactions were analyzed on a $15 \%$ SDS-PAGE gel and quantified as above. The quantitated ubiquitination was then plotted against the concentration of Cdh1 used for each reaction using the graphing program Prism, and the resulting data points analyzed with a hyperbolic curve fit using the equation $y=\left(\operatorname{Bmax}^{\star} X\right) /(\mathrm{Kd}+X)$. The $r$ values for the fits were 0.97 and 0.99 for wild-type and $c d c 27 \Delta$ APC, respectively.

For the experiments with C-box and IR mutant Cdh1 in Figure $5 \mathrm{D}$, wild-type, IP-AA, and IR $\Delta$ versions of Cdh1 were in vitro translated with equal efficiency (data not shown) and added in increasing amounts to APC reactions containing $5 \mathrm{nM}$ wild-type APC or $60 \mathrm{nM} c d c 27 \Delta \mathrm{APC}$ and ${ }^{125}$ I-labeled sea urchin cyclin fragment. Reactions proceeded for $20 \mathrm{~min}$ at room temperature before being run on 15\% SDS-PAGE gels and quantified as above. Quantitation was limited to those substrates with four or more ubiquitins, to avoid complications with substrate depletion in the wild-type APC reactions. The counts were normalized for enzyme levels.

\section{Acknowledgments}

We thank P. Hieter, A. Murray, A. Page, A. Rudner, and W. Seufert for antibodies and constructs, and members of the Toczyski and Morgan laboratories for helpful discussions and critical reading of the manuscript. This work was supported by grants from the National Institutes of Health to D.P.T. (GM070539) and D.O.M. (GM53270). M.E.M is supported by a Graduate Fellowship from the National Science Foundation.

\section{References}

Burton, J.L. and Solomon, M.J. 2001. D box and KEN box motifs in budding yeast Hsllp are required for APC-mediated degradation and direct binding to Cdc20p and Cdh1p. Genes \& Dev. 15: 2381-2395.

Burton, J.L., Tsakraklides, V., and Solomon, M.J. 2005. Assembly of an APC-Cdh1-substrate complex is stimulated by engagement of a destruction box. Mol. Cell 18: 533-542.

Carroll, C.W. and Morgan, D.O. 2002. The Doc1 subunit is a processivity factor for the anaphase-promoting complex. Nat. Cell Biol. 4: 880-887.

. 2005. Enzymology of the anaphase-promoting complex. Methods Enzymol. 398: 219-230.

Clarke, D.J., Segal, M., Andrews, C.A., Rudyak, S.G., Jensen, S., Smith, K., and Reed, S.I. 2003. S-phase checkpoint controls mitosis via an APC-independent Cdc20p function. Nat. Cell
Biol. 5: 928-935.

Gmachl, M., Gieffers, C., Podtelejnikov, A.V., Mann, M., and Peters, J.M. 2000. The RING-H2 finger protein APC11 and the E2 enzyme UBC4 are sufficient to ubiquitinate substrates of the anaphase-promoting complex. Proc. Natl. Acad. Sci. 97: 8973-8978.

Hilioti, Z., Chung, Y.S., Mochizuki, Y., Hardy, C.F., and CohenFix, O. 2001. The anaphase inhibitor Pds1 binds to the APC/ $\mathrm{C}$-associated protein Cdc20 in a destruction box-dependent manner. Curr. Biol. 11: 1347-1352.

Jaspersen, S.L., Charles, J.F., Tinker-Kulberg, R.L., and Morgan, D.O. 1998. A late mitotic regulatory network controlling cyclin destruction in Saccharomyces cerevisiae. Mol. Biol. Cell 9: 2803-2817.

Jaspersen, S.L., Charles, J.F., and Morgan, D.O. 1999. Inhibitory phosphorylation of the APC regulator Hctl is controlled by the kinase Cdc28 and the phosphatase Cdc14. Curr. Biol. 9: 227-236.

Kraft, C., Vodermaier, H.C., Maurer-Stroh, S., Eisenhaber, F., and Peters, J.M. 2005. The WD40 propeller domain of Cdh1 functions as a destruction box receptor for APC/C substrates. Mol. Cell 18: 543-553.

Kramer, E.R., Scheuringer, N., Podtelejnikov, A.V., Mann, M., and Peters, J.M. 2000. Mitotic regulation of the APC activator proteins CDC20 and CDH1. Mol. Biol. Cell 11: 15551569.

Leverson, J.D., Joazeiro, C.A., Page, A.M., Huang, H., Hieter, P., and Hunter, T. 2000. The APC11 RING-H2 finger mediates E2-dependent ubiquitination. Mol. Biol. Cell 11: 2315-2325.

Ohtoshi, A., Maeda, T., Higashi, H., Ashizawa, S., and Hatakeyama, M. 2000. Human p55(CDC)/Cdc20 associates with cyclin A and is phosphorylated by the cyclin A-Cdk2 complex. Biochem. Biophys. Res. Commun. 268: 530-534.

Passmore, L.A. 2004. The anaphase-promoting complex (APC): The sum of its parts? Biochem. Soc. Trans. 32: 724-727.

Passmore, L.A. and Barford, D. 2005. Coactivator functions in a stoichiometric complex with anaphase-promoting complex/ cyclosome to mediate substrate recognition. EMBO Rep. 6: 873-878.

Passmore, L.A., McCormack, E.A., Au, S.W., Paul, A., Willison, K.R., Harper, J.W., and Barford, D. 2003. Doc1 mediates the activity of the anaphase-promoting complex by contributing to substrate recognition. EMBO J. 22: 786-796.

Peters, J.M. 2002. The anaphase-promoting complex: Proteolysis in mitosis and beyond. Mol. Cell 9: 931-943.

Petroski, M.D. and Deshaies, R.J. 2005. Function and regulation of cullin-RING ubiquitin ligases. Nat. Rev. Mol. Cell. Biol. 6: 9-20.

Pfleger, C.M., Lee, E., and Kirschner, M.W. 2001. Substrate recognition by the Cdc20 and Cdh1 components of the anaphase-promoting complex. Genes \& Dev. 15: 2396-2407.

Pickart, C.M. and Eddins, M.J. 2004. Ubiquitin: Structures, functions, mechanisms. Biochim. Biophys. Acta 1695: 5572.

Prinz, S., Hwang, E.S., Visintin, R., and Amon, A. 1998. The regulation of $\mathrm{Cdc} 20$ proteolysis reveals a role for APC components Cdc23 and Cdc27 during S phase and early mitosis. Curr. Biol. 8: 750-760.

Puig, O., Caspary, F., Rigaut, G., Rutz, B., Bouveret, E., BragadoNilsson, E., Wilm, M., and Seraphin, B. 2001. The tandem affinity purification (TAP) method: A general procedure of protein complex purification. Methods 24: 218-229.

Rudner, A.D. and Murray, A.W. 2000. Phosphorylation by Cdc28 activates the Cdc20-dependent activity of the anaphase-promoting complex. J. Cell. Biol. 149: 1377-1390.

Schwab, M., Lutum, A.S., and Seufert. W. 1997. Yeast Hct1 is a 
Thornton et al.

regulator of Clb2 cyclin proteolysis. Cell 90: 683-693.

Schwab, M., Neutzner, M., Mocker, D., and Seufert, W. 2001. Yeast Hct1 recognizes the mitotic cyclin $\mathrm{Clb} 2$ and other substrates of the ubiquitin ligase APC. EMBO I. 20: 51655175 .

Schwickart, M., Havlis, J., Habermann, B., Bogdanova, A., Camasses, A., Oelschlaegel, T., Shevchenko, A., and Zachariae, W. 2004. Swm1/Apc13 is an evolutionarily conserved subunit of the anaphase-promoting complex stabilizing the association of Cdc16 and Cdc27. Mol. Cell. Biol. 24: 35623576.

Shirayama, M., Zachariae, W., Ciosk, R., and Nasmyth, K. 1998. The Polo-like kinase Cdc5p and the WD-repeat protein $\mathrm{Cdc} 20 \mathrm{p} /$ fizzy are regulators and substrates of the anaphase promoting complex in Saccharomyces cerevisiae. EMBO J. 17: 1336-1349.

Sikorski, R.S., Michaud, W.A., Wootton, J.C., Boguski, M.S., Connelly, C., and Hieter, P. 1991. TPR proteins as essential components of the yeast cell cycle. Cold Spring Harbor Symp. Quant. Biol. 56: 663-673.

Tang, Z., Li, B., Bharadwaj, R., Zhu, H., Ozkan, E., Hakala, K., Deisenhofer, J., and Yu, H. 2001. APC2 Cullin protein and APC11 RING protein comprise the minimal ubiquitin ligase module of the anaphase-promoting complex. Mol. Biol. Cell 12: 3839-3851.

Thornton, B.R. and Toczyski, D.P. 2003. Securin and B-cyclin/ CDK are the only essential targets of the APC. Nat. Cell. Biol. 5: 1090-1094.

Tung, J.J. and Jackson, P.K. 2005. Emil class of proteins regulate entry into meiosis and the meiosis I to meiosis II transition in Xenopus oocytes. Cell Cycle 4: 478-482.

Visintin, R., Prinz, S., and Amon, A. 1997. CDC20 and CDH1: A family of substrate-specific activators of APC-dependent proteolysis. Science 278: 460-463.

Vodermaier, H.C. 2004. APC/C and SCF: Controlling each other and the cell cycle. Curr. Biol. 14: R787-R796.

Vodermaier, H.C., Gieffers, C., Maurer-Stroh, S., Eisenhaber, F., and Peters, J.M. 2003. TPR subunits of the anaphase-promoting complex mediate binding to the activator protein CDH1. Curr. Biol. 13: 1459-1468.

Wendt, K.S., Vodermaier, H.C., Jacob, U., Gieffers, C., Gmachl, M., Peters, J.M., Huber, R., and Sondermann, P. 2001. Crystal structure of the APC10/DOC1 subunit of the human anaphase-promoting complex. Nat. Struct. Biol. 8: 784-788.

Willems, A.R., Schwab, M., and Tyers, M. 2004. A hitchhiker's guide to the cullin ubiquitin ligases: SCF and its kin. Biochim. Biophys. Acta 1695: 133-170.

Yoon, H.J., Feoktistova, A., Wolfe, B.A., Jennings, J.L., Link, A.J., and Gould, K.L. 2002. Proteomics analysis identifies new components of the fission and budding yeast anaphasepromoting complexes. Curr. Biol. 12: 2048-2054.

$\mathrm{Yu}, \mathrm{H} .2002$. Regulation of APC-Cdc20 by the spindle checkpoint. Curr. Opin. Cell. Biol. 14: 706-714.

Yu, H., Peters, J.M., King, R.W., Page, A.M., Hieter, P., and Kirschner, M.W. 1998. Identification of a cullin homology region in a subunit of the anaphase-promoting complex. Science 279: 1219-1222.

Zachariae, W., Shin, T.H., Galova, M., Obermaier, B., and Nasmyth, K. 1996. Identification of subunits of the anaphasepromoting complex of Saccharomyces cerevisiae. Science 274: 1201-1204.

Zachariae, W., Schwab, M., Nasmyth, K., and Seufert, W. 1998a. Control of cyclin ubiquitination by CDK-regulated binding of Hctl to the anaphase promoting complex. Science 282: $1721-1724$.
Zachariae, W., Shevchenko, A., Andrews, P.D., Ciosk, R., Galova, M., Stark, M.J., Mann, M., and Nasmyth, K. 1998b. Mass spectrometric analysis of the anaphase-promoting complex from yeast: Identification of a subunit related to cullins. Science 279: 1216-1219.

Zheng, N., Schulman, B.A., Song, L., Miller, J.J., Jeffrey, P.D., Wang, P., Chu, C., Koepp, D.M., Elledge, S.J., Pagano, M., et al. 2002. Structure of the Cul1-Rbx1-Skp1-F boxSkp2 SCF ubiquitin ligase complex. Nature 416: 703-709. 


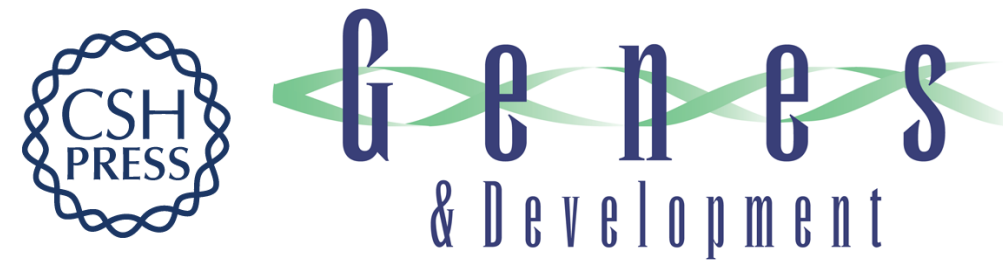

\section{An architectural map of the anaphase-promoting complex}

Brian R. Thornton, Tessie M. Ng, Mary E. Matyskiela, et al.

Genes Dev. 2006, 20:

Access the most recent version at doi:10.1101/gad.1396906

Supplemental http://genesdev.cshlp.org/content/suppl/2006/02/01/20.4.449.DC1
Material

References This article cites 43 articles, 18 of which can be accessed free at: http://genesdev.cshlp.org/content/20/4/449.full.html\#ref-list-1

License

Email Alerting Receive free email alerts when new articles cite this article - sign up in the box at the top Service right corner of the article or click here.

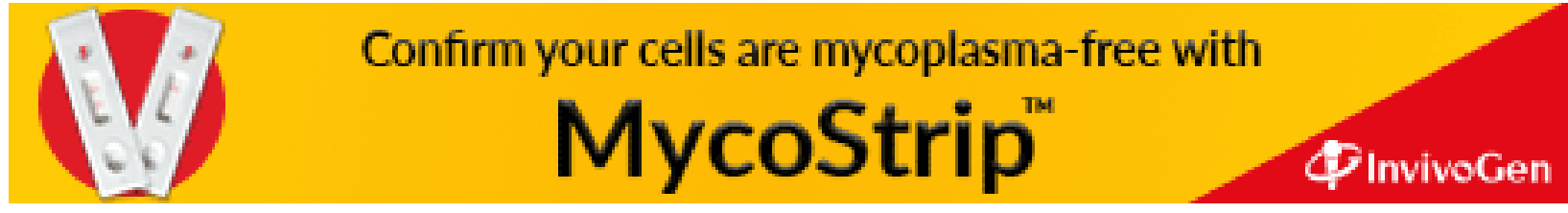

